\title{
Experimental Identification and Control of a Cantilever Beam Using ERA/OKID with a LQR Controller
}

\author{
L. A. Gagg F. • S. M. da Conceição • C. H. Vasques • \\ G. L. C. M. de Abreu • V. Lopes Jr. • M. J. Brennan
}

Received: 31 August 2012 / Revised: 26 March 2013 / Accepted: 25 December 2013 / Published online: 11 March 2014

(C) Brazilian Society for Automatics-SBA 2014

\begin{abstract}
This paper presents an experimental study of the system identification and vibration control of a cantilever beam. For system identification, a white noise was applied, and the response signal was measured. These signals were used to feed the eigensystem realization algorithmObserver/Kalman Filter identification method. The identified system was reduced using the Hankel norm model. An linear quadratic regulator controller was projected to operate just on the first two natural frequencies of the structure. The damping ratio of the first mode was effectively increased from 0.009 to 0.046 .
\end{abstract}

Keywords Optimal control - Linear quadratic regulator . ERA · OKID

\section{Introduction}

A relatively new area of engineering is that of smart structures, in which actuators and sensors are incorporated onto a structure to enable it to adapt to its environment. One application area of importance is the active control of vibrations (Gatti et al. 2007; Zhang et al. 2008). This is of concern in this paper, particularly, the active vibration control of a cantilever beam.

Initially, to implement active control, a dynamic model of the mechanical system is required. A worthy model is

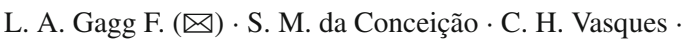

G. L. C. M. de Abreu • V. Lopes Jr. · M. J. Brennan

GMSINT - Grupo de Materiais e Sistemas Inteligentes,

UNESP/FEIS - Faculdade de Engenharia de Ilha Solteira,

Av. Brasil, 56, Ilha Solteira, SP CEP 15385-000, Brazil

e-mail: luizarthur.gagg@gmail.com

URL: http://www.dem.feis.unesp.br/gmsint that one whose mathematical representation is able to make a good prediction of the behavior of the system in time and space. The process of achieving this representation from observations and experimental data is called identification (Ljung 1987). In this field, the good intuition of the researcher is of the most value (Santo 2001; Deistler 2004). One of the first identification and modeling methods is called Least Squares Method and was developed by Gauss in 1795 (Monteiro 2006). (Juang and Pappa 1985) developed an identification method, which is convenient for this purpose. This method makes possible to represent the system in terms of its state variables. The algorithm used in this case, which was initially called the eigensystem realization algorithm (ERA), has had two extensions. One of these is the ERA/DC (Juang and Wright 1988), where DC abbreviation means "data correlation", and the other is the ERA/OKID (Juang and Pappa 1985), where OKID means Observer/Kalman Filter. The latter method was designed to be used mainly in the identification of lightly damped structures. Originally, this technique was conceived for the identification of modal parameters and reduction of dynamic models. However, the ERA can be used as a powerful tool for controller design if incorporated, for instance, with a linear quadratic regulator (LQR). Nowadays, the ERA and/or the ERA/OKID are widely used in the aerospace sector (Lim et al. 1998), vibratory mechanical systems (Alves and Ribeiro 2004), large civil construction (Lus et al. 2002), and automotive vehicle structures (Rezende and Borges 2004).

The purpose of this paper is to assess the ERA/OKID algorithm with an LQR controller through an experiment on a cantilever beam, whereupon the control actuator and sensor were piezoelectric materials bonded on the structure. The aim was to actively increase the damping so that the free vibration of the beam decayed away much more quickly. 


\section{Problem Definition}

The problem consists in two parts: system identification and then its reduction by the Hankel norm model, and the controller design. The formulations of these steps are described in this section.

\subsection{Eigensystem Realization Algorithm}

To identify or achieve the realization of a system, it is required to evaluate whether this system can or cannot be controlled and/or observed. Of particular concern is the concept of the minimal realization of a system (Ho and Kalman 1965). Consider a linear system and time invariant. It can be described by the discrete time equations:

$$
\begin{aligned}
\mathbf{x}(k+1) & =\mathbf{A} \mathbf{x}(k)+\mathbf{B u}(k) \\
\mathbf{y}(k) & =\mathbf{C x}(k)+\mathbf{D u}(k)
\end{aligned}
$$

where $\mathbf{x}(k) \in \mathfrak{R}^{n}$ is the state vector; $\mathbf{u}(k) \in \mathfrak{R}^{r}$ is the control system signal vector; $k$ represents a discrete time step; $\mathbf{A} \in$ $\mathfrak{R}^{n x n}$ and $\mathbf{B} \in \mathfrak{R}^{n x r}$ are the constant dynamic system matrices containing the physical parameters of the system; $\mathbf{C}$ and $\mathbf{D}$ are the output and feedthrough matrices, respectively; and $\mathbf{y}$ is the vector of outputs (Dorf and Bishop 2001). Applying an unit impulse input $\mathbf{u}=\left[\begin{array}{llll}1 & 0 & 0 & \ldots\end{array}\right]$ and considering $\mathbf{x}(0)=0$ (Alves and Ribeiro 2004), it is possible to obtain a recursive expression to the response $\mathbf{Y}$ as shown below:

for $k=0$

$$
\begin{aligned}
& \mathbf{x}(1)=\mathbf{A} \mathbf{x}(0)+\mathbf{B u}(0)=\mathbf{B} \\
& \mathbf{y}(0)=\mathbf{C x}(0)+\mathbf{D u}(0)=\mathbf{D} \\
& \text { for } k=k \\
& \qquad \mathbf{x}(k+1)=\mathbf{A x}(k)+\mathbf{B u}(k)=\mathbf{A}^{k} \mathbf{B} \\
& \qquad \mathbf{y}(k)=\mathbf{C x}(k)+\mathbf{D u}(0)=\mathbf{C A}^{k-1} \mathbf{B}=\mathbf{Y}_{k}
\end{aligned}
$$

which results in the following sequence of matrices known as Markov parameters matrices

$$
\mathbf{Y}_{0}=\mathbf{D}, \mathbf{Y}_{1}=\mathbf{C B}, \mathbf{Y}_{2}=\mathbf{C A B}, \ldots, \mathbf{Y}_{k}=\mathbf{C A}^{k-1} \mathbf{B}
$$

Therefore, the Markov parameters are the output of the system with an impulse input and are obtained experimentally assembling the following matrix:

$$
\mathrm{Y}_{k}=\left[\begin{array}{cccc}
\mathrm{y}_{k}^{(1,1)} & \mathrm{y}_{k}^{(1,2)} & \cdots & \mathrm{y}_{k}^{(1, j)} \\
\mathrm{y}_{k}^{(2,1)} & \mathrm{y}_{k}^{(2,2)} & \cdots & \mathrm{y}_{k}^{(2, j)} \\
\vdots & \vdots & \ddots & \vdots \\
\mathrm{y}_{k}^{(i, 1)} & \mathrm{y}_{k}^{(i, 2)} & \cdots & \mathrm{y}_{k}^{(i, j)}
\end{array}\right]_{m x r}
$$

where $m$ is the number of outputs and $r$ is the number of inputs, $i=1,2, \ldots, m$ and $j=1, \ldots, r$. Each column represents the response of the system to the input $j$ and each row represents the output $i$. With these parameters the Hankel matrix $\mathbf{H}$ can be determined using Eq. (4)

$$
\begin{aligned}
& \mathrm{H}(k-1) \\
& =\left[\begin{array}{cccc}
\mathrm{Y}_{k} & \mathrm{Y}_{k+1} & \cdots & \mathrm{Y}_{k}+\beta-1 \\
\mathrm{Y}_{k+1} & \mathrm{Y}_{k+2} & \cdots & \mathrm{Y}_{k}+\beta \\
\vdots & \vdots & \ddots & \vdots \\
\mathrm{Y}_{k}+\alpha-1 & \mathrm{Y}_{k}+\alpha & \cdots & \mathrm{Y}_{k}+\alpha+\beta-2
\end{array}\right]
\end{aligned}
$$

where the choice of the parameters $\alpha$ and $\beta$ is carried out by empirical method and depends on the experience of the designer. This matrix can be decomposed into its singular values, so that for $k=1$ :

$\mathbf{H}(0)=\mathbf{R} \sum \mathbf{S}^{\mathrm{T}}$

where the matrices $\mathbf{R}$ and $\mathbf{S}$ are orthonormal and $\Sigma$ is a rectangular diagonal matrix assembled by the singular values.

The matrices that describe the realization in state space are obtained by Alves (2005)

$$
\begin{aligned}
& \hat{\mathbf{A}}=\sum_{n}^{-1 / 2} \mathbf{R}_{n}^{\mathbf{T}} \mathbf{H}(1) \mathbf{S}_{n} \sum_{n}^{-1 / 2} \\
& \hat{\mathbf{B}}=\sum_{n}^{1 / 2} \mathbf{S}_{n}^{\mathrm{T}} \mathbf{E}_{m} \\
& \hat{\mathbf{C}}=\mathbf{E}_{r} \mathbf{R}_{n} \sum_{n}^{1 / 2} \\
& \hat{\mathbf{D}}=\mathbf{Y}_{0}
\end{aligned}
$$

where $\mathbf{E}_{r}$ and $\mathbf{E}_{m}$ are matrices composed of identity matrices and matrices of zeros. The superscript $\mathrm{T}$ indicates the transpose of the matrix. The subscripts $m, n$, and $r$ are the number of outputs, the order of the system, and the number of inputs, respectively, and indicate that the matrices are truncated versions of their original. The hat on the matrix indicates that the matrix is the identified one. The subscript 0 in $\mathbf{Y}_{0}$ indicates that the Markov parameter $\mathbf{Y}$ corresponds to time step $k$ equal to zero.

\subsection{Model Reduction}

Basically, a problem about models reduction consists in finding, for a high-order model, linear, stable, and time-invariant $\mathbf{H}(k)$, a new model with a reduced order $\mathbf{H}_{\mathrm{g}}(k)$, so that the difference of performance between both models, at the same circumstances, can be as low as possible. The model order is its dimension in state space or the degree of the denominator polynomial of the transfer function. This difference of performance could be quantified through the $\mathrm{H}_{2}$ and $\mathrm{H}_{\infty}$ norms (Skogestad and Postlethwaite 2005). 


\subsubsection{Reduction Through Hankel Norm}

In this approach, the problem is processed as follows: given a model $\mathbf{H}(k)$ of $n$-order, find a reduced $g$-order model, $\mathbf{H}_{g}(k)$, in a way where the approximation error of the Hankel Norm, $\left\|\mathbf{H}(k)-\mathbf{H}_{\mathrm{g}}(k)\right\|_{\mathrm{H}}$, is minimized. The Hankel Norm of any stable transfer function $\mathbf{E}(k)$ is defined as (Gawronski 1998):

$$
\|\mathbf{E}(k)\|_{H}=\max _{i} \sigma
$$

where $\sigma$ is the largest Hankel singular value. The Hankel singular values are defined by:

$\sigma_{i}=\sqrt{\lambda_{i}\left(\mathbf{W}_{\mathbf{c}} \mathbf{W}_{\mathbf{o}}\right)}$

where $\mathbf{W}_{\mathbf{c}}$ and $\mathbf{W}_{\mathbf{o}}$ are the gramians of controllability and observability of $\mathbf{E}(k)$, respectively, and $\lambda_{i}$ are the eigenvalues of $\left(\mathbf{W}_{\mathbf{c}} \mathbf{W}_{\mathbf{o}}\right)$. Therefore, in this optimization, it is attempted to find an error that is somehow close to be entirely nonobservable and entirely non-controllable.

The problem of reduction through Hankel Norm has been considered among several authors. A bound to the Infinite Norm is particularly interesting because it is better than the bounds to the truncated balancing and to the balanced residualization. The theorem below (Skogestad and Postlethwaite 2005) yields a proceeding to a reduction of models for stable and square transfer functions through Hankel Norm:

Theorem: consider $\mathbf{H}(k)$ as a stable and square transfer function with the singular values of Hankel $\sigma_{1} \geq \sigma_{2} \geq \cdots \geq$ $\sigma_{\mathrm{g}} \geq \sigma_{\mathrm{g}+1} \geq \sigma_{\mathrm{g}+2}=\sigma_{\mathrm{g}+3}=\cdots=\sigma_{\mathrm{g}+1}>\sigma_{\mathrm{g}+1+1} \geq$ $\cdots \geq \sigma_{\mathrm{n}}>0$, thus a reduced model of $g$-order, $\mathbf{H}_{\mathrm{g}}(k)$, can be created.

Consider (A, B, C, D) as a balanced realization of $\mathbf{H}(k)$ with singular values of Hankel rearranged so that the gramian is:

$$
\begin{aligned}
\sum & =\operatorname{diag}\left(\sigma_{1}, \sigma_{2}, \ldots, \sigma_{\mathrm{g}}, \sigma_{\mathrm{g}+1+1}, \ldots, \sigma_{\mathrm{n}}, \sigma_{\mathrm{g}+1}, \sigma_{\mathrm{g}+1}\right. \\
& =\operatorname{diag}\left(\sum_{1}, \sigma_{\mathrm{g}+1} \mathbf{I}\right)
\end{aligned}
$$

where $\mathbf{I}$ is the identity matrix in appropriate dimensions. Partitioning $(\mathbf{A}, \mathbf{B}, \mathbf{C}, \mathbf{D})$ according to $\sum$

$A=\left[\begin{array}{ll}A_{11} & A_{12} \\ A_{21} & A_{22}\end{array}\right] B=\left[\begin{array}{l}B_{1} \\ B_{2}\end{array}\right] C=\left[\begin{array}{ll}C_{1} & C_{2}\end{array}\right]$

Defining ( $\hat{\mathbf{A}}, \hat{\mathbf{B}}, \hat{\mathbf{C}}, \hat{\mathbf{D}})$ as (Skogestad and Postlethwaite 2005):

$$
\begin{aligned}
& \hat{\mathbf{A}}=\Gamma^{-1}\left(\sigma_{k+1}^{2} \mathbf{A}_{11}^{\mathrm{T}}+\sum_{1} \mathbf{A}_{11} \sum_{1}-\sigma_{k+1} \mathbf{C}_{1}^{\mathrm{T}} \mathbf{U} \mathbf{B}_{1}^{\mathrm{T}}\right) \\
& \hat{\mathbf{B}}=\Gamma^{-1}\left(\sum_{1} \mathbf{B}_{1}+\sigma_{k+1} \mathbf{C}_{1}^{T} \mathbf{U}\right) \\
& \left.\hat{\mathbf{C}}=\mathbf{C}_{1} \sum_{1}+\sigma_{k+1} \mathbf{U} \mathbf{B}_{1}^{\mathrm{T}}\right) \\
& \hat{\mathbf{D}}=\mathbf{D}-\sigma_{k+1} \mathbf{U}
\end{aligned}
$$

$$
1
$$

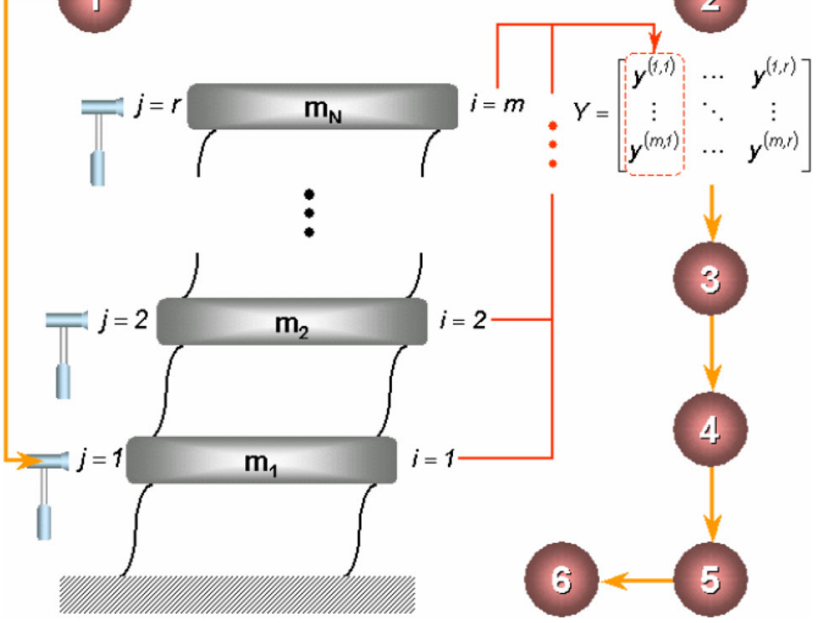

Fig. 1 ERA flowchart. Font: Alves (2005)

where $\mathbf{U}$ is a unitary matrix satisfying $\mathbf{B}_{2}=\mathbf{C}_{1}^{\mathrm{T}} \mathbf{U}$ and $\Gamma=$ $\sum_{1}^{2}-\sigma_{k+1}^{2} \mathbf{I}$. The matrix $\hat{\mathbf{A}}$ has "stable" eigenvalues (all of them have negative real part); the other $n-g$ eigenvalues have positive real part. Therefore,

$\mathbf{H}_{g}(k)+\mathbf{F}(k)=\left[\begin{array}{cc}\hat{\mathbf{A}} & \hat{\mathbf{B}} \\ \hat{\mathbf{C}} & \hat{\mathbf{D}}\end{array}\right]$

here, $\mathbf{H}_{\mathrm{g}}(k)$ is the stable approximation of $g$-order of $\mathrm{H}(k)$, and $\mathbf{F}(k)$ is the non-stable transfer function (all the poles have a positive real part) of $n-g-1$ order. Hankel Norm of the error between $\mathbf{H}(k)$ and $\mathbf{H}_{\mathrm{g}}(k)$ is equal to the $(k+1)$-th singular value of $\mathbf{H}(\mathrm{g})$ 's Hankel, i.e.,

$\left\|\mathbf{H}(k)-\mathbf{H}_{\mathrm{g}}(k)\right\|_{\mathrm{H}}=\sigma_{g+1}(\mathbf{H})$

\subsection{ERA Algorithm}

The ERA flowchart is shown by Figs. 1 and 2 and its algorithm can be summed up by the following steps:

Step 1: Acquire all the outputs for each input when this one is excited by an impulse signal.

Step 2: With the step 1 completely done, it is possible to assemble the matrix of Eq. (3).

Step 3: Construct the Hankel matrix represented by Eq. (4). The parameters $\alpha$ and $\beta$ should be estimated. Also, in this step, the order of the system, $n$, must be estimated.

Step 4: Take the Hankel matrix $\mathbf{H}(0)$ and decompose in singular values.

Step 5: Take the Hankel matrix $\mathbf{H}(1)$ and apply it in Eqs. (6)-(9) to get the realization. 


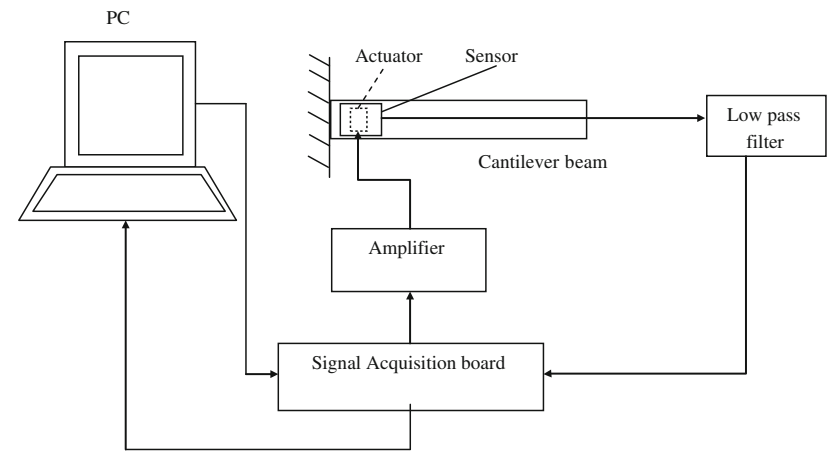

Fig. 2 Experimental setup

Step 6: If necessary, do a new identification and return to step 5 with a new order or reduce the system through Hankel Norm.

Step 7: Compare the impulsive responses and the frequency response functions (FRFs) of the real and identified system.

\subsection{Identification Using an Observer/ Kalman Filter}

When the free vibration of a structure, excited by an impulse, has a very small decay rate, there is a large computational effort when the ERA method is applied (Alves 2005). However, a state observer can be added, which introduces artificial damping in the system reducing the length of the data vector acquired. This method is called OKID (Phan et al. 1992). Mathematically, the system turns into another one, where the Markov parameters are obtained from the observer. The OKID method recovers the Markov parameters of the real system and the gain of the observer. One of the great advantages of this method is the ability to obtain the impulse response of the real system from any kind of input signal (Bernal and Gunes 2000), which can then feed the ERA algorithm.

The following mathematical deductions were taken from Alves (2005). Adding and subtracting the term $\mathbf{G y}(k)$ to the right-hand side of the equation of state in Eq. (1), it results in

$\mathbf{x}(k+1)=\mathbf{A x}(k)+\mathbf{B u}(k)+\mathbf{G y}(k)-\mathbf{G y}(k)$

$\mathbf{y}(k)=\mathbf{C x}(k)+\mathbf{D u}(k)$

So, replacing the first $\mathbf{y}(k)$ in $\mathbf{x}(k+1)$ the equation becomes:

$\mathbf{x}(k+1)=\mathbf{A x}(k)+\mathbf{B u}(k)+\mathbf{G}[\mathbf{C x}(k)+\mathbf{D u}(k)]-\mathbf{G y}(k)$

or

$\mathbf{x}(k+1)=\mathbf{A x}(k)+\mathbf{B u}(k)+\mathbf{G C x}(k)+\mathbf{G D u}(k)-\mathbf{G y}(k)$

Bringing together $\mathbf{x}(k)$ and $\mathbf{y}(k)$, it is reached:

$\mathbf{x}(k+1)=(\mathbf{A}+\mathbf{G C}) \mathbf{x}(k)+(\mathbf{B}+\mathbf{G D}) \mathbf{u}(k)-\mathbf{G y}(k)$ and this equation can be written as:

$\mathbf{x}(k+1)=\overline{\mathbf{A}} \mathbf{x}(k)+\overline{\mathbf{B}} \mathbf{v}(k)$

where

$\overline{\mathbf{A}}=\mathbf{A}+\mathbf{G C} ; \quad \overline{\mathbf{B}}=[\mathbf{B}+\mathbf{G D}-\mathbf{G}] ; \mathbf{v}=\{\mathbf{u}(k) \mathbf{y}(k)\}^{\mathrm{T}}$

Considering zero as initial conditions, the following sequence can be shown:

$$
\begin{aligned}
\mathbf{x}(k+1)= & \overline{\mathbf{A}} \mathbf{x}(k)+\overline{\mathbf{B}} \mathbf{v}(k) \\
\mathbf{x}(k+2)= & \overline{\mathbf{A}} \mathbf{x}(k+1)+\overline{\mathbf{B}} \mathbf{v}(k+1) \\
= & \overline{\mathbf{A}}^{2} \mathbf{x}(k)+\overline{\mathbf{A}} \overline{\mathbf{B}} \mathbf{v}(k)+\overline{\mathbf{B}} \mathbf{v}(k+1) \\
\mathbf{x}(k+3)= & \overline{\mathbf{A}} \mathbf{x}(k+2)+\overline{\mathbf{B}} \mathbf{v}(k+2) \\
= & \overline{\mathbf{A}}^{3} \mathbf{x}(k)+\overline{\mathbf{A}}^{2} \overline{\mathbf{B}} \mathbf{x}(k) \\
& +\overline{\mathrm{A}} \overline{\mathbf{B}} \mathbf{v}(k+1)+\overline{\mathbf{B}} \mathbf{v}(k+2) \\
\mathbf{x}(k+p)= & \overline{\mathbf{A}} \mathbf{x}(k+p+1)+\mathbf{B} \mathbf{v}(k+p+1) \\
= & \overline{\mathbf{A}}^{p} \mathbf{x}(k)+\overline{\mathbf{A}}^{p-1} \overline{\mathbf{B}} \mathbf{v}(k)+\mathbf{A}^{p-2} \overline{\mathbf{B}} \mathbf{v}(k+1)+\cdots \\
& +\overline{\mathbf{B}} \mathbf{v}(k+p-1)
\end{aligned}
$$

and using Eq. (18), the following relation is built:

$$
\begin{aligned}
\mathbf{y}(k+p)= & \mathbf{C x}(k+p)+\mathbf{D u}(k+p) \\
= & \mathbf{C} \overline{\mathbf{A}}^{\mathrm{p}_{\mathbf{x}}(k)+\mathbf{C A}} \overline{\mathrm{p}}^{-1} \overline{\mathbf{B}} v(k) \\
& +\mathbf{C A}^{p-2} \overline{\mathbf{B}} \mathbf{v}(k+1)+\ldots \\
& +\mathbf{C} \overline{\mathbf{B}}^{p-2} \mathbf{v}(k+p-1)+\mathbf{D u}(k+p)
\end{aligned}
$$

The set of the Eqs. (20) and (21) can be written for a sequence of $k=0,1, \ldots, l-1$ as:

$\mathbf{y}=\mathbf{C} \overline{\mathbf{A}}^{p} \mathbf{x}+\overline{\mathbf{Y}} \overline{\mathbf{V}}$

where

$\mathbf{x}=[\mathbf{x}(0) \mathbf{x}(1) \cdots \mathbf{x}(\ell-p-2)]$,

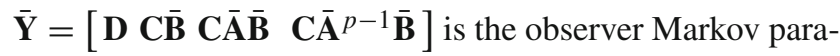
meters,

$\overline{\mathbf{v}}=\left[\begin{array}{cccc}\mathbf{u}(p) & \mathbf{u}(p+1) & \cdots & \mathbf{u}(\ell-1) \\ \mathbf{v}(p-1) & \mathbf{v}(p) & \cdots & \mathbf{v}(\ell-2) \\ \mathbf{v}(p-2) & \mathbf{v}(p-1) & \cdots & \mathbf{v}(\ell-3) \\ \vdots & \vdots & \ddots & \vdots \\ \mathbf{v}(0) & \mathbf{v}(1) & \cdots & \mathbf{v}(\ell-p-1)\end{array}\right]_{r(p+1) x(\ell-p)}$

$l$ is the number of acquired data. The choice of $p$ determines the number of the observer Markov parameters. When $\overline{\mathbf{A}}^{p}$ is small enough and all the states are limited, the Eq. (22) can be approximated to:

$\overline{\mathbf{y}}=\overline{\mathbf{Y}} \overline{\mathbf{V}}$

where $\overline{\mathbf{y}}=[\mathbf{y}(p) \mathbf{y}(p+1) \cdots \mathbf{y}(\ell-1)]$. The observer Markov parameters can be calculated by solving the Eq. (23) by least mean square as shown next.

$\overline{\mathbf{Y}}=\overline{\mathbf{y}} \overline{\mathbf{V}}^{\dagger}\left[\overline{\mathbf{V}} \overline{\mathbf{V}}^{\dagger}\right]^{-1}$ 
The symbol † indicates the pseudoinverse.

\subsubsection{System Markov Parameters}

To recover the system Markov parameters in $\mathbf{Y}$ from the observer Markov parameters, the following partition is necessary.

$\overline{\mathbf{Y}}=\left[\begin{array}{lllll}\overline{\mathbf{Y}}_{0} & \overline{\mathbf{Y}}_{1} & \overline{\mathbf{Y}}_{2} & \cdots & \overline{\mathbf{Y}}_{p}\end{array}\right]$

where

$$
\begin{aligned}
\overline{\mathbf{Y}}_{0} & =\mathrm{D} \\
\overline{\mathbf{Y}}_{k} & =\mathbf{C} \overline{\mathbf{A}}^{k-1} \overline{\mathbf{B}} \\
& =\left[\mathbf{C}(\mathbf{A}+\mathbf{G C})^{k-1}(\mathbf{B}+\mathbf{G D})-\mathbf{C}(\mathbf{A}+\mathbf{G C})^{k-1} \mathbf{G}\right] \\
& \triangleq\left[\overline{\mathbf{Y}}_{k}^{(1)}-\overline{\mathbf{Y}}_{k}^{(2)}\right] ; \quad k=1,2,3, \ldots
\end{aligned}
$$

It is important to say that each observer Markov parameter has one portion, $\overline{\mathbf{Y}}_{k}^{(1)}$, related to the input and another one, $\overline{\mathbf{Y}}_{k}^{(2)}$, related to the output. In order to discover the system Markov parameters the first Markov parameter of $\mathbf{Y}_{1}$ is taken. It is possible to make the following deductions:

$\mathbf{Y}_{1}=\mathbf{C B}=\mathbf{C}(\mathbf{B}+\mathbf{G D})-(\mathbf{C G}) \mathbf{D}=\overline{\mathbf{Y}}_{1}^{(1)}-\overline{\mathbf{Y}}_{1}^{(2)} \mathbf{D}$

To obtain $\mathbf{Y}_{2}=\mathbf{C A B}$, first consider the product $\overline{\mathbf{Y}}_{2}^{(1)}$

$$
\begin{aligned}
\overline{\mathbf{Y}}_{2}^{(1)} & =\mathbf{C}(\mathbf{A}+\mathbf{G C})(\mathbf{B}+\mathbf{G D}) \\
& =\mathbf{C A B}+\mathbf{C G C B}+\mathbf{C}(\mathbf{A}+\mathbf{G C}) \mathbf{G D} \\
& =\mathbf{Y}_{2}+\overline{\mathbf{Y}}_{2}^{(1)} \mathbf{Y}_{1}-\overline{\mathbf{Y}}_{2}^{(2)} \mathbf{D}
\end{aligned}
$$

Isolating $\mathbf{Y}_{2}$ in Eq. (31), it is reached:

$\mathbf{Y}_{2}=\overline{\mathbf{Y}}_{2}^{(1)}-\overline{\mathbf{Y}}_{2}^{(1)} \mathbf{Y}_{1}+\overline{\mathbf{Y}}_{2}^{(2)} \mathbf{D}$

Doing the same for $\overline{\mathbf{Y}}_{3}^{(1)}$

$$
\begin{aligned}
\overline{\mathbf{Y}}_{3}^{(1)}= & \mathbf{C}(\mathbf{A}+\mathbf{G C})^{2}(\mathbf{B}+\mathbf{G D}) \\
= & \mathbf{C}\left(\mathbf{A}^{2}+\mathbf{G C A}+\mathbf{A G C}+\mathbf{G C G C}\right)(\mathbf{B}+\mathbf{G D}) \\
= & \mathbf{C A}^{2} \mathbf{B}+\mathbf{C G C A B}+\mathbf{C}(\mathbf{A}+\mathbf{G C}) \mathbf{C G B} \\
& +\mathbf{C}(\mathbf{A}+\mathbf{G C})^{\mathbf{2}} \mathbf{G D} \\
= & \mathbf{Y}_{3}+\overline{\mathbf{Y}}_{1}^{(2)} \mathbf{Y}_{2}+\overline{\mathbf{Y}}_{2}^{(2)} \mathbf{Y}_{1}+\overline{\mathbf{Y}}_{3}^{(2)} \mathbf{D}
\end{aligned}
$$

Isolation $\mathrm{Y}_{3}$ yields:

$$
\mathrm{Y}_{3}=\overline{\mathbf{Y}}_{3}^{(1)}-\overline{\mathbf{Y}}_{1}^{(2)} \mathrm{Y}_{2}-\overline{\mathbf{Y}}_{2}^{(2)} \mathrm{Y}_{1}-\overline{\mathbf{Y}}_{3}^{(2)} \mathrm{D}
$$

The other Markov parameters can be determined using this logic as follows:

$$
\begin{aligned}
& \mathbf{Y}_{k}=\overline{\mathbf{Y}}_{k}^{(1)}-\sum_{i=1}^{k} \overline{\mathbf{Y}}_{i}^{(2)} \mathbf{Y}_{k-i} \quad \text { for } \quad k=1, \ldots, p \\
& \mathbf{Y}_{k}=-\sum_{i=1}^{p} \overline{\mathbf{Y}}_{i}^{(2)} \mathbf{Y}_{k-i} \text { for } \quad k=p+1, \ldots, \infty
\end{aligned}
$$

Once the system Markov parameters are obtained, the Hankel matrix must be built and then the matrices $\mathbf{A}, \mathbf{B}, \mathbf{C}, \mathbf{D}$ can be found applying the ERA algorithm.

\subsubsection{Observer Gain Markov Parameters}

To recover the observer gain Markov parameters, it is necessary to consider the following sequence:

$\mathbf{Y}_{k}^{0}=\mathbf{C A}^{k-1} \mathbf{G}$

where the overwritten ${ }^{0}$ means a relation with the observer gain. The first Markov parameter of the observer gain is just:

$\mathbf{Y}_{1}^{0}=\mathrm{CG}=\mathrm{Y}_{1}^{(2)}$

The next is taken considering $\overline{\mathbf{Y}}_{2}^{(2)}$ as:

$$
\begin{aligned}
\overline{\mathbf{Y}}_{2}^{(2)} & =\mathbf{C A} \mathbf{A}=\mathbf{C}(\mathbf{A}+\mathbf{G C}) \mathbf{G}=\mathbf{C A G}+\mathbf{C G C G} \\
& =\mathbf{Y}_{2}^{0}+\overline{\mathbf{Y}}_{1}^{(2)} \mathbf{Y}_{1}^{0}
\end{aligned}
$$

Isolating $\mathbf{Y}_{2}^{0}$ in Eq. (39), it is reached:

$\mathbf{Y}_{2}^{0}=\overline{\mathbf{Y}}_{2}^{(2)}-\overline{\mathbf{Y}}_{1}^{(2)} \mathbf{Y}_{1}^{0}$

Doing the same considering $\overline{\mathbf{Y}}_{3}^{(2)}$ as:

$$
\begin{aligned}
\overline{\mathbf{Y}}_{3}^{(2)}= & \mathbf{C} \overline{\mathbf{A}}^{2} \mathbf{G} \\
= & \mathbf{C}(\mathbf{A}+\mathbf{G C})^{2} \mathbf{G}=\mathbf{C}\left(\mathbf{A}^{2}+\mathbf{A G C}\right. \\
& +\mathbf{G C A}+\mathbf{C G C G}) \mathbf{G} \\
= & \mathbf{C}\left[\mathbf{A}^{2}+(\mathbf{A}+\mathbf{G C}) \mathbf{G C}+\mathbf{G C A}\right] \mathbf{G} \\
= & \mathbf{C A}^{2} \mathbf{G}+\mathbf{C A} \mathbf{A} \mathbf{C G}+\mathbf{C G C A G} \\
= & \mathbf{Y}_{3}^{0}+\overline{\mathbf{Y}}_{2}^{(2)} \mathbf{Y}_{1}^{0}+\overline{\mathbf{Y}}_{1}^{(2)} \mathbf{Y}_{2}^{0}
\end{aligned}
$$

Again, isolating $\mathbf{Y}_{3}^{0}$, it yields:

$\mathbf{Y}_{3}^{0}=\overline{\mathbf{Y}}_{3}^{(2)}-\overline{\mathbf{Y}}_{2}^{(2)} \mathbf{Y}_{1}^{0}-\overline{\mathbf{Y}}_{1}^{(2)} \mathbf{Y}_{2}^{0}$

Therefore, the next general relationship can be deduced as:

$\mathbf{Y}_{1}^{0}=\mathbf{C G}=\overline{\mathbf{Y}}_{1}^{(2)}$

$\mathbf{Y}_{k}^{0}=\overline{\mathbf{Y}}_{k}^{(2)}-\sum_{i=1}^{k-1} \overline{\mathbf{Y}}_{\mathrm{i}}^{(2)} \mathbf{Y}_{k-\mathrm{i}}^{0}$ for $k=2, \ldots, p$

$\mathbf{Y}_{k}^{0}=\overline{\mathbf{Y}}_{k}^{(2)}-\sum_{i=1}^{k-1} \overline{\mathbf{Y}}_{\mathrm{i}}^{(2)} \mathbf{Y}_{k-\mathrm{i}}^{0}$ for $k=p+1, \ldots, \infty$

\subsubsection{ERA/OKID Algorithm}

The ERA/OKID algorithm can be summed up by the following steps: 
Step 1: Choose the value of $p$, which determines the observer Markov parameters. Build the matrices $\overline{\mathbf{y}}$ and $\overline{\mathbf{V}}$ of Eq. (23).

Step 2: Calculate the observer Markov parameters using Eq. (24) by least squares.

Step 3: Get the Markov parameters of the system, Eqs. (35) and (36), and the Markov parameters of the observer gain, using Eq. (43).

Step 4: With the Markov parameters of the system, apply the ERA algorithm.

\subsection{LQR Controller}

In modern control theory, the LQR controller has an important place. This controller ensures excellent stability margins and good disturbance rejection (Ogata 2003). The method allows the adoption of a performance index, which can optimize the response of the system. The LQR controller is based on the minimization of a quadratic criterion, which is associated with the power of the state variables and control signals. Consider the system to be controlled, which is described by Eq. (1). The quadratic optimal problem is determining the constant matrix $\mathbf{K}$ of the control vector in

$\mathbf{u}=\mathbf{K x}$

The matrix $\mathbf{K} \in \Re^{m \times n}$ must be determined to minimize the performance index of the system given by

$J=\sum_{k=0}^{\infty}\left(\mathbf{x}^{\mathrm{T}}(k) \mathbf{Q} \mathbf{x}(k)+\mathbf{u}^{\mathrm{T}}(k) \mathbf{R} \mathbf{u}(k)\right)$

where $\mathbf{Q}$ is a real symmetric positive definite matrix (or positive semi-definite) and $\mathbf{R}$ is a real symmetric positive definite matrix. The $\mathbf{Q}$ and $\mathbf{R}$ matrices are typically chosen to be diagonal matrices. It is necessary to solve the Riccati matrix Equation given by

$\mathbf{A}^{\mathrm{T}}\left(\mathbf{P}-\mathbf{P B}\left(\mathbf{R}+\mathbf{B}^{\mathrm{T}} \mathbf{P B}\right)^{-1} \mathbf{B}^{\mathrm{T}} \mathbf{P}\right) \mathbf{A}+\mathbf{Q}-\mathbf{P}=\mathbf{0}$

for a positive $\mathbf{Q}$ to determine the elements of matrix $\mathbf{K}$ which minimizes the performance index. The matrix of gains $\mathbf{K}$ can be found from

$\mathbf{K}=\left(\mathbf{R}+\mathbf{B}^{\mathrm{T}} \mathbf{P A}\right)^{-1} \mathbf{B}^{\mathrm{T}} \mathbf{P A}$

\section{Experimental Work}

The techniques described in Sect. 2 were applied to identify the state-space matrices $(\mathbf{A}, \mathbf{B}, \mathbf{C}, \mathbf{D})$ of the aluminum cantilever beam with dimensions $35.4 \times 42 \times 2.0 \mathrm{~mm}$, Figs. 3 and 4 . The system was excited by random noise using a Lead-Zirconate-Titanate (PZT) actuator, Fig. 5, with dimensions: $42 \times 23 \times 0.2 \mathrm{~mm}$. The vibration was measured using a Polyvinylidene-fluoride (PVDF) patch, Fig. 6, of dimensions

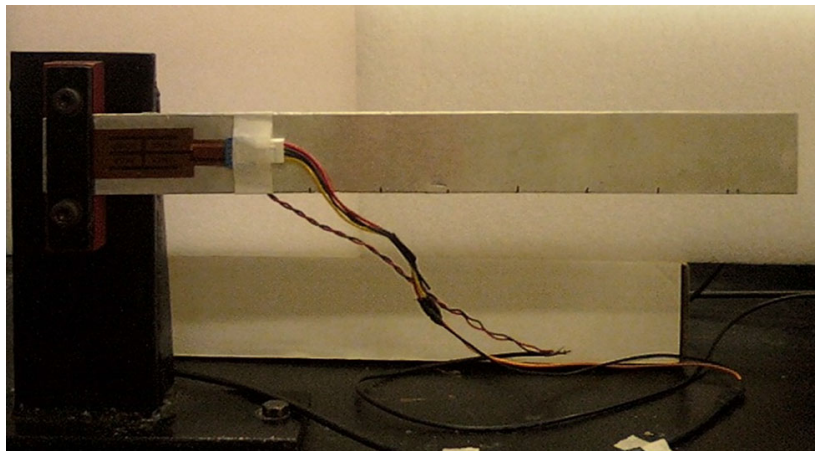

Fig. 3 Cantilever beam

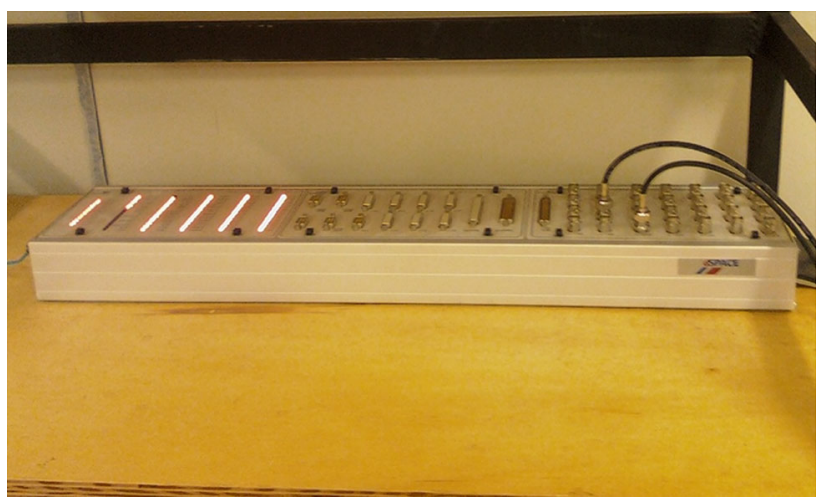

Fig. 4 Acquisition board

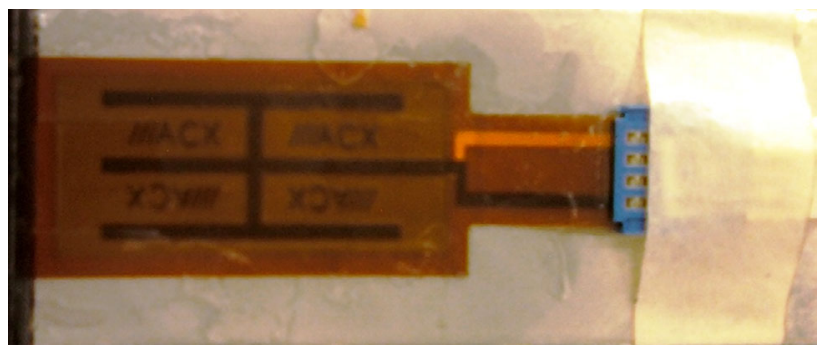

Fig. 5 PZT

$30.0 \times 10.0 \times 0.2 \mathrm{~mm}$ positioned at the root of the cantilever as shown in Fig. 2.

The DSpace data acquisition board, Fig. 4, was used to convert the analog signal into a digital signal. In this direction, the gain of the board is 0.1 . When the signal that leaves the computer is converted into an analogical signal, it is amplified by a factor of 10 by the board. The amplifier, Fig. 7, was configured to yield a gain of 20 in the analogical signal that leaves the acquisition board. A low-pass filter, Fig. 8, with a cut-off frequency of $100 \mathrm{~Hz}$ was used to attenuate unwanted high frequencies.

The experiment was divided into two parts: the first was identifying a dynamic model of the cantilever beam using the ERA/OKID algorithm; the second was designing an LQR controller based on the system identified. 


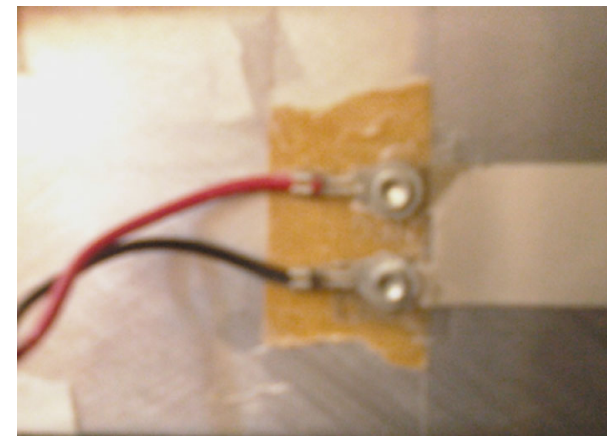

Fig. 6 PVDF

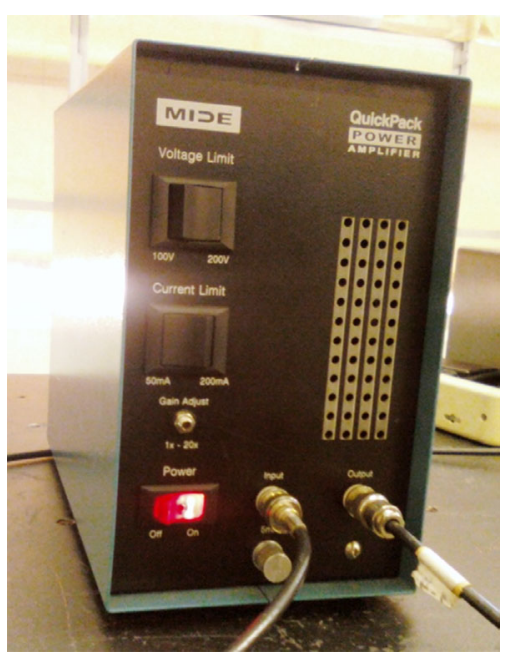

Fig. 7 Amplifier

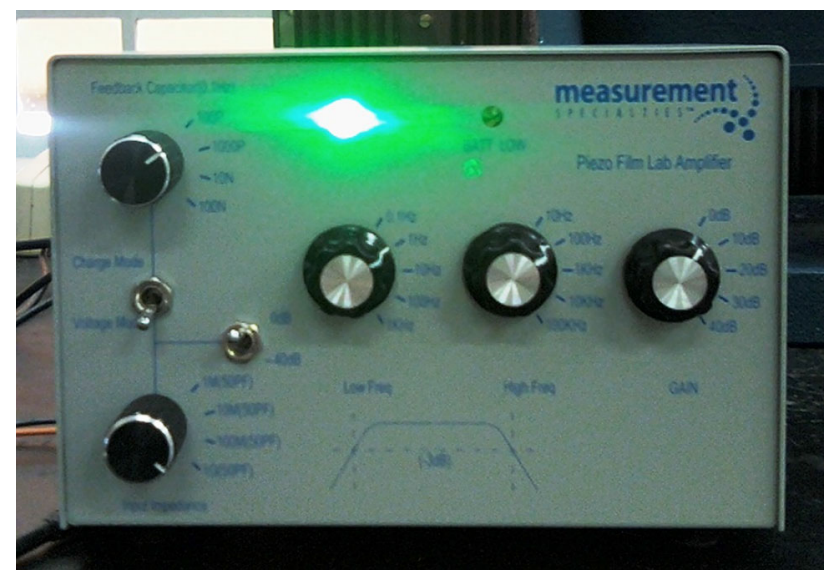

Fig. 8 Filter

\subsection{Experimental Realization}

It is known that to perform the system realization with the ERA/OKID algorithm any kind of input signal can be used (Alves 2005). In this experiment, it was utilized a white noise, which is basically a signal with random properties. For this kind of voltage (input signal), the beam has vibrated in an indertermined mode. The software Dspace Control Desk was employed to display the input and output signal in real time. This software allows to save a file .mat, which afterwards can be opened as struct in the Matlab ${ }^{\circledR}$. With the possession of the signals, it is possible to feed the algorithm ERA/OKID. The sample rate adopted was $1,000 \mathrm{~Hz}$ and 5 samples of $5 \mathrm{~s}$ to each one were used. The Figs. 9 and 10 below compare the input signal distribution and the output signal distribution with the Normal distribution, respectively.

These Figures demonstrate a good agreement between the considered signals distribution, input and output, with the Normal distribution. Thus, it can be verified that the white noise utilized in this paper has a normal distribution or, at least, is close to it. Moreover, it can be inferred that the output signal has a relation with the input signal.

To know if the input and output signals are well related, the coherence analysis must be done. The coherence is a function of frequency with values between 0 and 1 and denotes the relation between two signals for each value of frequency. The closer to the unity, the better the coherence between the signals. Coherence is calculated as the ratio of the module of the spectral density square of the signals' crossed power, $\mathbf{S}_{\mathbf{u y}}$, by the product of the power spectral of both input signals, $\mathbf{S}_{\mathbf{u}}$, and output, $\mathbf{S}_{\mathbf{y}}$, that is,

$\gamma_{y u}^{2}(f)=\frac{\left|\mathbf{S}_{\mathbf{u y}}\right|^{2}}{\mathbf{S}_{\mathbf{u}} \mathbf{S}_{\mathbf{y}}}$

where $f$ is the frequency.

The coherence is illustrated in Fig. 11.

By analyzing the Fig. 11, it can be observed that the input and output are well related up to approximately $450 \mathrm{~Hz}$. For higher frequencies, the relationship is not so good. Probably this occurs due to the intensification of nonlinearities.

The implementation of the algorithm ERA/OKID was made by (Alves 2005), who provided it to this work. Some parameters of this implementation must be set particularly for this experiment. Among them, there are, the reduced system state number $(g)$, the system order or the system state number $(n)$, and the Markov parameters number $(p)$. These parameters were chosen arbitrarily and were tested with a lot of combinations of values aiming to get the best approximation between the reduced system without the observer and the real system without the observer. It was verified that the reduced system state number should be as large as possible to ensure a good approximation. The identification of the reduced system can be directly performed to eliminate the model reduction step. Nevertheless, in this case, the rank of the observability and controllability matrix would decrease, and the approximation between the identified system and the real system would not be satisfactory. Therefore, it is better to identify 
Fig. 9 Input signal

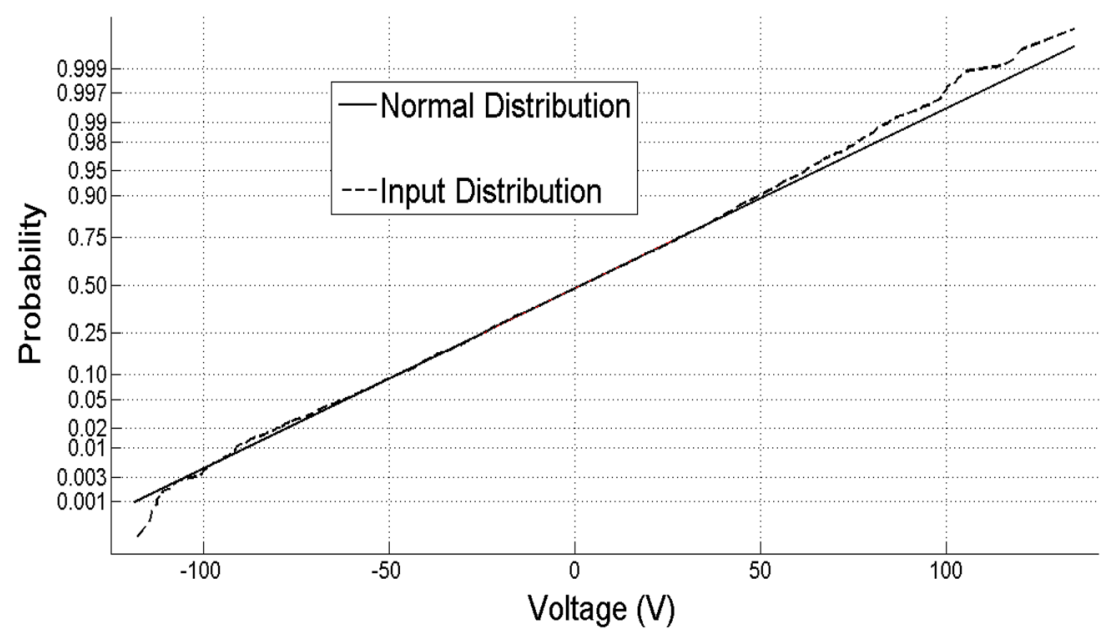

Fig. 10 Output signal

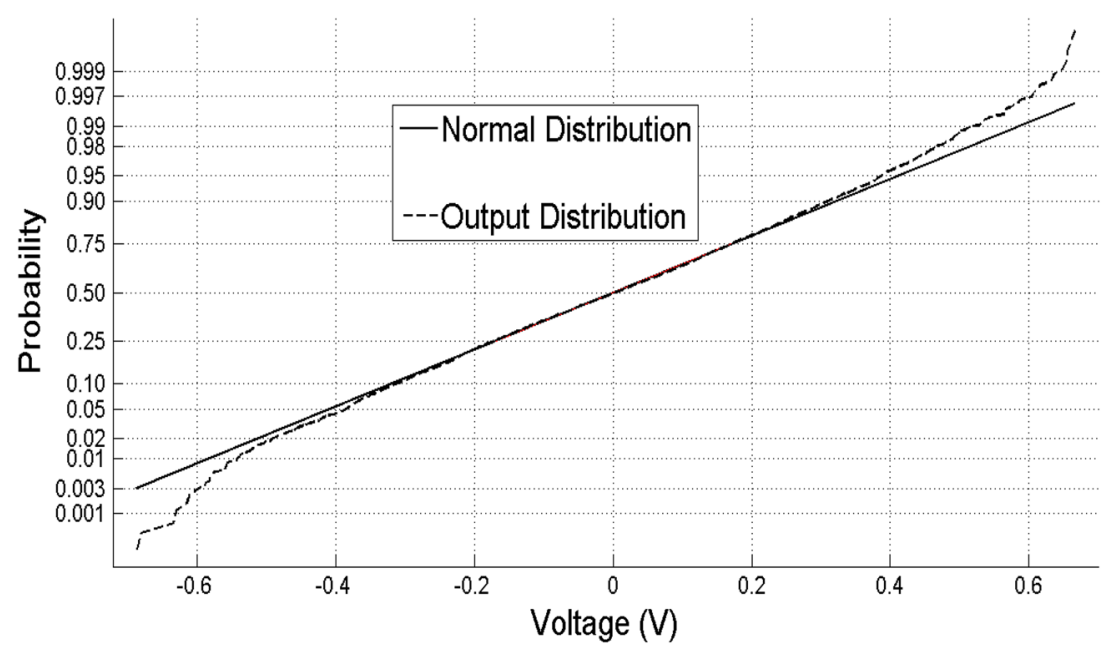

Fig. 11 Coherence

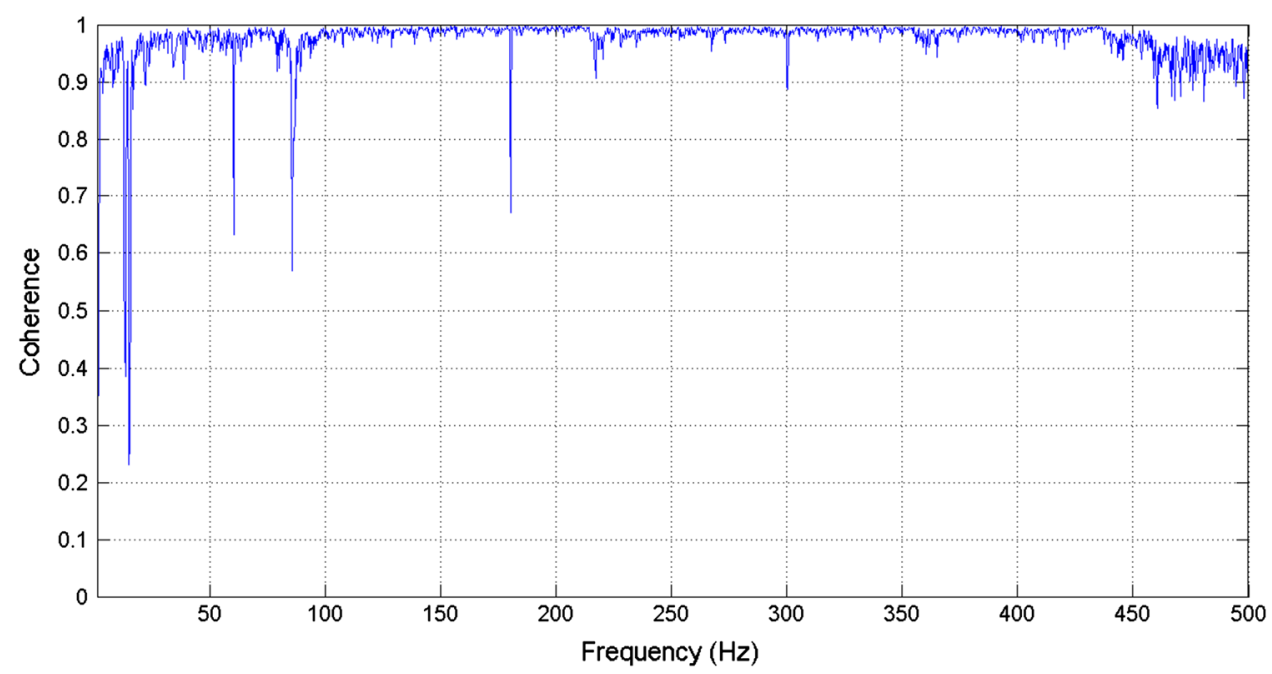

a higher order system to elevate this rank and apply a model reduction to obtain a reduced system with the order value equal to the rank of observability and controllability matrix because the largest possible state dimension of the reduced system is equal to this rank. Thus, the highest value for this parameter in this experiment was 5. If a system has one degree 
Fig. 12 Comparison between the FRF's a magnitude, $\mathbf{b}$ phase

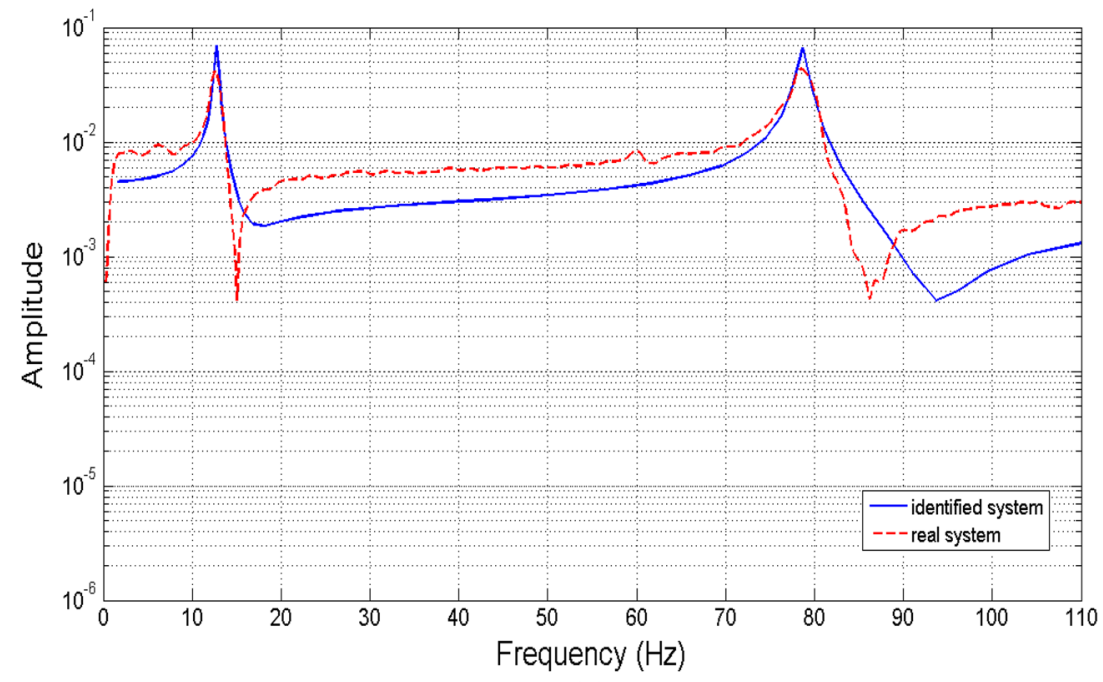

(a)

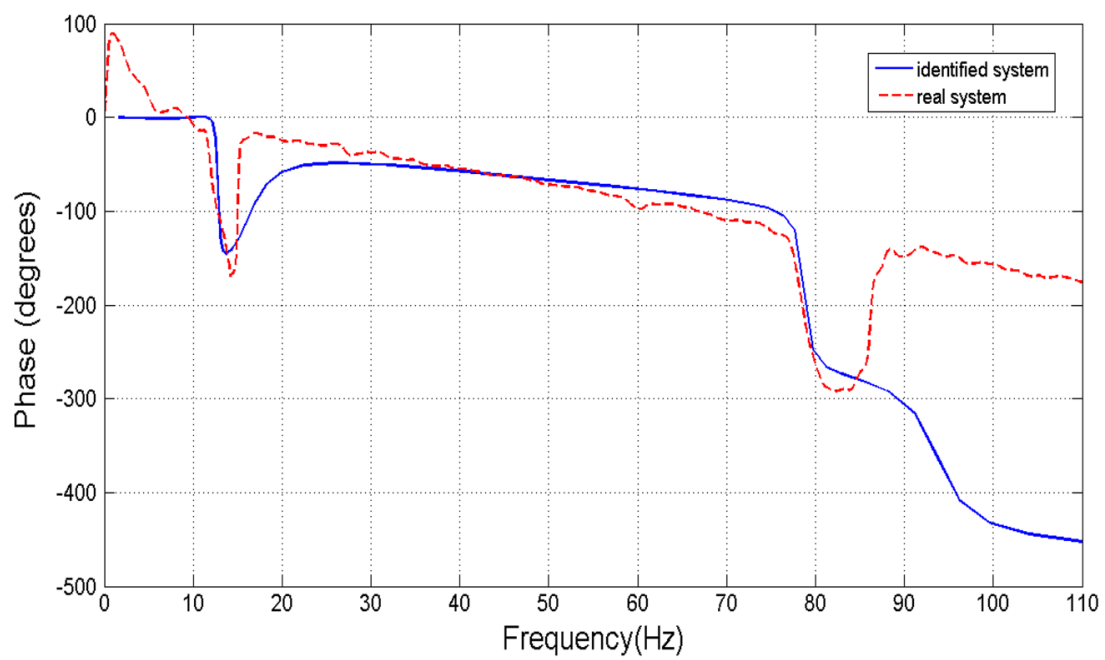

(b) of freedom, the order of this system in state space would be two. For each degree of freedom added to this system, two orders are added to the state space representation. So, the value 5 of the reduced system order in this work implies that it is possible to observe and control just the first two degrees of freedom.

For the other parameters, the best combination chosen was, 30 for the system state number and 40 for Markov parameters number. Always remember that the Markov parameters number must be at least equal to the system state number. This combination allows the identification of a model with controllability and observability of the two first vibration modes. A smaller combination has presented the controllability and observability of the first mode only, and in a bigger combination computational modes without physical meaning have appeared.
Figure 12 compares the FRF of the real system with the identified system for the first two modes of vibration. The real system was calculated using the Matlab command etfe. Actually, this command estimates an empirical transfer function as reason between the Fourier transform of the system response and the Fourier transform of the signal input employing the fast fourier transform (FFT).

Once the Markov parameters of the system were obtained, the ERA algorithm was applied using the Hankel norm model technique (Gawronski 2004) to determine the following system matrices according to Eqs. (6)-(9):

$$
\mathbf{A}=\left[\begin{array}{ccccc}
-642.8849 & -14.8770 & -13.6454 & -45.8243 & 28.4181 \\
0 & -1.0231 & 80.4548 & -1.7811 & 3.5774 \\
0 & -79.9607 & -1.2743 & -1.3411 & 2.2735 \\
0 & 0 & 0 & -1.5745 & 496.9992 \\
0 & 0 & 0 & -491.8237 & -5.1919
\end{array}\right]
$$




$$
\begin{aligned}
& \mathbf{B}=\left[\begin{array}{lllll}
-1.8218 & -0.3777 & 0.1237 & -0.3028 & 0.6066
\end{array}\right]^{\mathrm{T}} \\
& \mathbf{C}=\left[\begin{array}{lllll}
-1.7962 & -0.0058 & 0.3621 & 0.1825 & -0.5290
\end{array}\right] \\
& \mathbf{D}=-0.0026
\end{aligned}
$$

\subsection{Controller Project}

As soon as the matrices were known, it was possible to design a controller to reduce the free vibration of the cantilever beam. MATLAB has the command lqr that solves the Ricatti equation, Eq. (46), and determines the optimal retroaction matrix $\mathbf{K}$, Eq. (47). As the matrix $\mathbf{R}$ is associated with the magnitude of the control signal, this must be chosen to the voltage of the control signal not to exceed a critical value. In the experiment, the input voltage was applied to the PZT actuator, which had a critical voltage of approximately $180 \mathrm{~V}$. Therefore, to check the voltage level input, a numerical simulation was carried out in the MATLAB ${ }^{\circledR}$ Toolbox simulink.

As the modeled system consists of five state variables, and PVDF can capture only one signal, it is necessary to use a state observer to estimate the other states. Actually, the other four states could never be measured because they do not have physical meaning. The estimation of the states was made in closed loop. The observer was determined using

$\mathbf{e}(k+1)=(\mathbf{A}-\mathbf{L C}) \mathbf{e}(k)$

where $\mathbf{e}$ is the error vector between the measured output and the estimated output calculated through the state vector estimated by the observer and $\mathbf{L}$ is the matrix of the observer gains. If the eigenvalues of $(\mathbf{A}-\mathbf{L} \mathbf{C})$ have negative real parts, the error tends to zero. In the experiment, the gain $\mathbf{L}$ was calculated using the MATLAB command place. The dynamic of the observer must be faster than the system dynamic, thus the eigenvalues of the observer were chosen with high negative real parts as shown next:

eig $=\left[\begin{array}{lll}-100-150-200-250-300\end{array}\right]$

where eig is the eigenvalues of $(\mathbf{A}-\mathbf{L C})$. The matrices obtained for the LQR controller and for the observer were

$$
\begin{aligned}
\mathbf{K} & =\left[\begin{array}{lllll}
-0.0070 & -0.7646 & 0.2499 & -0.2103 & 0.4414
\end{array}\right] \\
\mathbf{L} & =\left[\begin{array}{lllll}
-6.5738 & 35.7409 & 62.1362 & -180.2308 & -655.6986
\end{array}\right]^{\mathrm{T}}
\end{aligned}
$$

The matrix $\mathbf{R}$ is actually a scalar, and was chosen to be 0.2 and the matrix $\mathbf{Q}$ was chosen as an identity matrix of order 5. Figure 13 shows the simulation of the controller action together with the observer on the beam.

It is important to point out that the state observer developed in the state estimation does not have relation with the state observer utilized in the system realization, this last has the main function of introducing an artificial damping in the system. Figure 13 also shows a gain of 20 in the control signal. This gain was added to simulate the amplifier gain. It

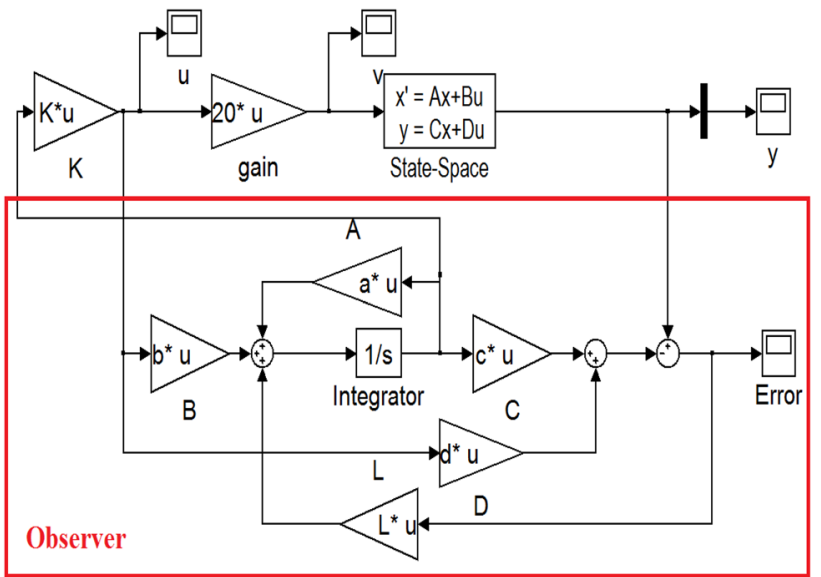

Fig. 13 Illustration of the state observer in the simulation in simulink of the controller action on the beam

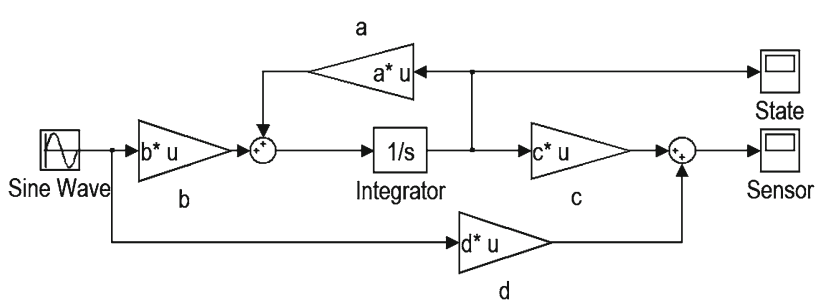

Fig. 14 Block diagram to get the initial conditions

can be observed that it was utilized for continuous blocks like "1/s" in Fig. 13. This can be done because the solver used was the ode1, which contains the Euler method. This solver works in the continuous mode, but when simulink is executed, the control loop is discretized to fixed step, for this case, the step is 0.001 seconds. On the order hand, it can be discretized the controller for the discrete time 0.001 seconds using the command $c 2 d$ and using discrete blocks for the integrator. The results would be the same.

To set the initial conditions in the simulation, the following procedure has been carried out. It was verified that the fundamental frequency of the reduced system was $12.76 \mathrm{~Hz}$. So, the system was excited by a sine wave with this frequency to force a condition close enough to the resonance. Thereby all the state variables should be destabilized with significant amplitudes after a time interval. The last state in this time interval was captured and set as initial condition. Figure 14 illustrates the block diagram of this procedure.

The intention is simulating the controller in extreme conditions. The initial conditions captured were

$\mathbf{I}_{\mathrm{c}}=\left[\begin{array}{lllll}-0.4025 & -4.7369 & 10.4490 & 0.1342 & 0.0461\end{array}\right]^{\prime}$

Figure 15 shows the behavior of the voltage sent to PZT during the simulation. 
Fig. 15 Behavior of the voltage sent to PZT in the simulation

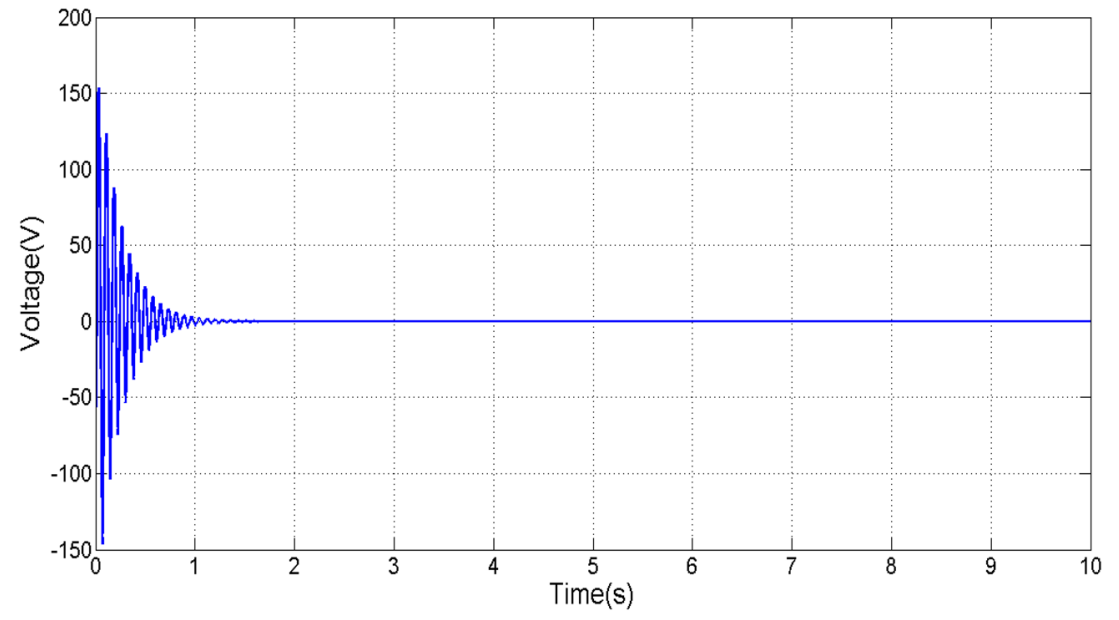

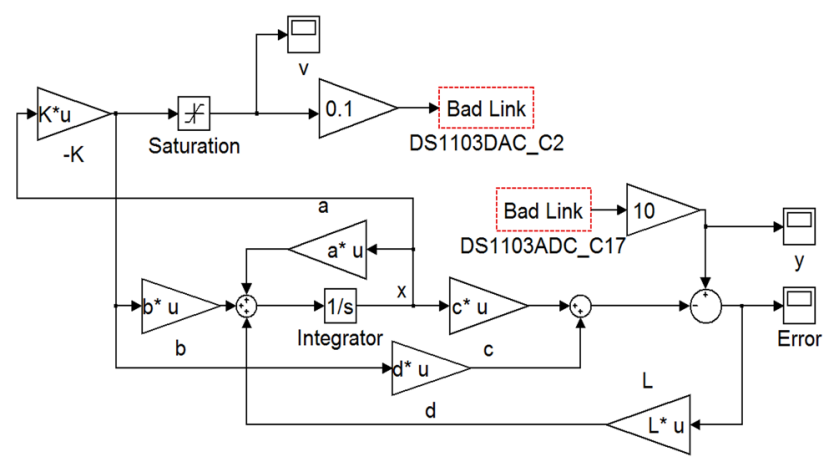

Fig. 16 Experimental block diagram

If this voltage is greater than $180 \mathrm{~V}$, the biggest value of $\mathbf{R}$ must be chosen. In the case, the value of 0.2 keeps safe the PZT and closes the controller project.

\subsection{Experimental Test}

Figure 16 shows the experiment block diagram with the action of the LQR controller on the beam

Again it was utilized the Euler method for the solver. The saturation block was configured to cut off voltages above $7.5 \mathrm{~V}$ and below $-7.5 \mathrm{~V}$. This ensures the health of the PZT. With this set, the highest voltage coming out from the saturation block is $7.5 \mathrm{~V}$, which is multiplied by 0.1 to compensate the gain of 10 of the dSpace board. As the amplifier has a gain of 20, the highest value of the voltage that reaches the PZT is $150 \mathrm{~V}$. When the voltage generated by the PVDF is sent to the acquisition board, it is converted into a digital signal with a 0.1 gain. Thus, it is necessary to set a gain block of 10 to compensate it. After understanding the experimental block diagram, the experiment itself can be done. The software dSPACE ControlDesk is open to exhibit the input and output signals of the system.
The first test was conducted without controller action: the operator provided a close impulsive input with his own finger at the edge of the beam which made it oscillate freely. The controller was then turned on and, again, the operator provided another close impulsive force with his own finger, roughly equal to the first touch, at the same edge. The comparison between the uncontrolled vibration and the controlled vibration is shown in Fig. 17.

The control signal is illustrated in Fig. 18.

\section{Discussion}

From the results, it can be observed that the experimental method of identifying ERA/OKID is effective, as is the LQR controller in suppressing the free vibrations of the cantilever beam. The performance of the control signal during the simulation is evident in Fig. 15, where it is observed that the input signal reached a peak of about $150 \mathrm{~V}$ ensuring the physical integrity of the actuator. Afterward it was observed that it is safe to perform the experiment. It is noted that the uncontrolled system took about $14 \mathrm{~s}$ to decay away, whereas the controlled system took only about $2.5 \mathrm{~s}$ in Fig. 17.

The choice of the number of Markov parameters and the estimated order of the physical system are arbitrary, and so the experience and intuition of the designer are important. A fact observed due to the different combinations of these parameters is that they are related to physical system estimated order, $n$. The higher this value is, the better the approximation of the FRF's with the physical system will be, thus, it is quite feasible to choose a high value of $n$, since it is provided the reduction model tool.

The identification method ERA/OKID coupled with an LQR controller has some disadvantages. The mainly one is the impossibility of identifying an instable system because all the formulation has considered a stable system. If an iden- 
Fig. 17 Comparison between the uncontrolled vibration and the controlled vibration
Fig. 18 Voltage sent to the PZT before amplifying
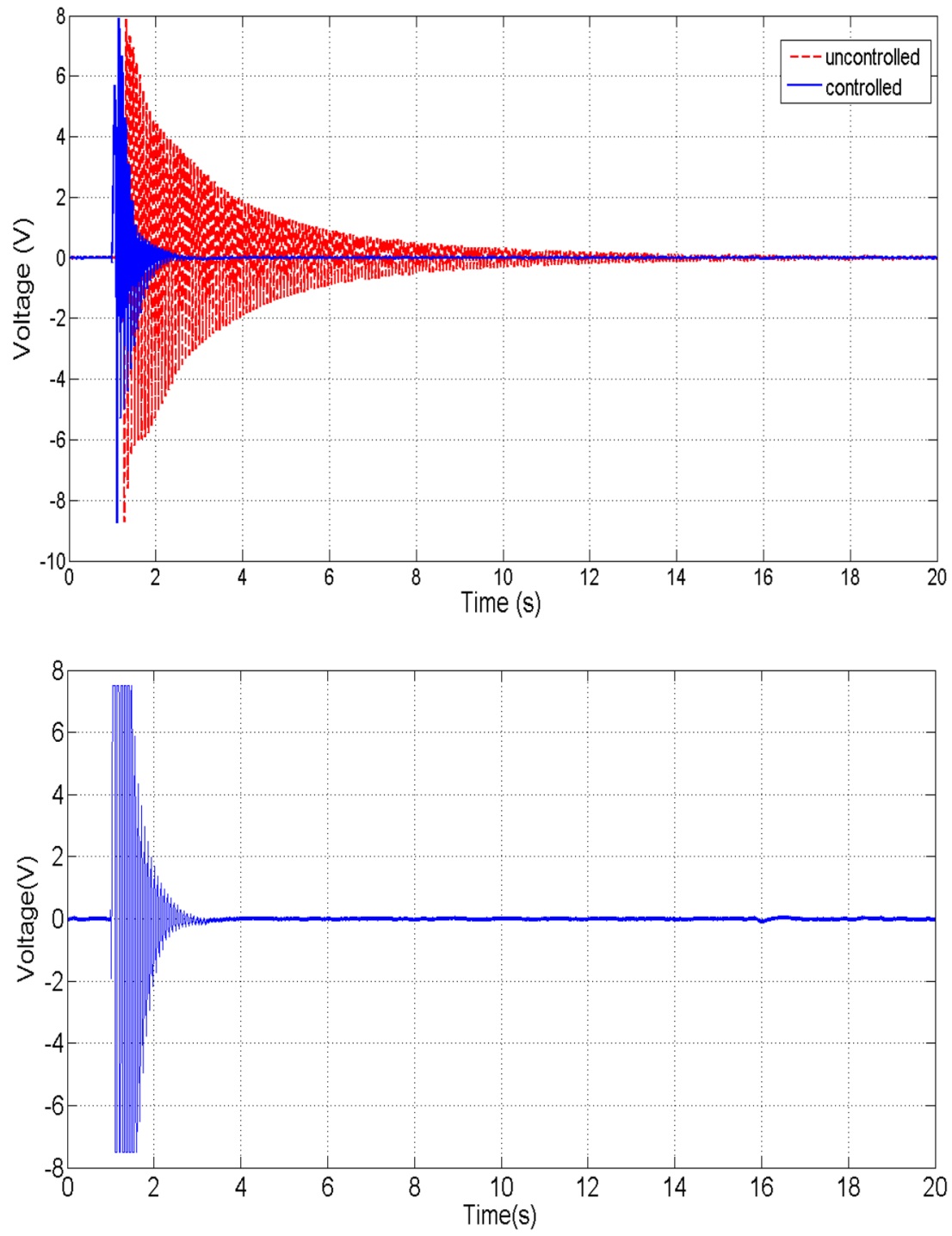

tification of an instable system was made, it is quite probably that the FRFs of the identified and real systems would not be similar, so that the controller projected would not present the desired robustness or simply would not work.

\section{Conclusions}

This paper has described an experimental study upon the control of a cantilever beam. LQR control was implemented, which required a state-space model of the system. This was achieved using the ERA/OKID system identification technique with the beam being driven by random noise. The subsequent model was reduced to a fifth-order model. An impulsive force was applied to the tip of the beam, and the controller, which sensed the vibration using a PVDF patch at the root of the beam, applied a control force through a PZT actuator connected on the beam. And it has significantly reduced the time for the transient vibration to decay away. The damping ratio of the first mode was increased from 0.009 to 0.046 according to logarithmic decrement method demonstrating the efficacy of the control strategy.

One purpose to future work would be a study of the capability of ERA/OKID algorithm to identify nonlinearities. To highly nonlinear systems, would it be possible to identify a physical system to project a classic controller, as the LQR, with the desire robustness?

Another purpose derives from the fact that this work has some non-automated steps for instance, the choice of the parameters $n, P, \alpha$, and $\beta$. This procedure could be automated using a genetic algorithm. In this case, an initial population for these parameters has to be chosen so the GA algorithm optimizes the population through an objective function. This one could be the distance between the real and the identified FRFs. Thus, a method to evaluate this distance must be chosen, which is theoretically simple. 
Acknowledgments The authors would like to acknowledge the support of the Instituto Nacional de Ciência e Tecnologia - Estruturas Inteligentes em Engenharia (INCT).

\section{References}

Alves, M. T. S. (2005). Avaliação numérica e experimental dos métodos ERA e ERA/OKID para a identificação de sistemas mecânicos. Dissertation of Master's Degree, UFU-MG, School of Mechanical Engineering, Uberlândia.

Alves, M. T. S., \& Ribeiro, J. F. (2004). Identificação de Sistemas Mecânicos Lineares usando o ERA (eigensystem realization algorithm). Belém, PA: III Congresso Nacional de Engenharia Mecânica.

Bernal, D., Gunes, B. (2000). Performance of an observer state-space identification in the presence of mild nonlinearities. In Proc. 2000 American control conference, Chicago, IL.

Deistler, M. (2004). System identification-general aspects and structure, interuniversity attraction poles-phase $V$-dynamical systems and control: Computation, identification and modelling. Louvainla-Neuve.

Dorf, R. C., \& Bishop, R. H. (2001). Sistemas de controle modernos. Tradução Bernardo Severo da Silva Filho. Rio de Janeiro: LTC, c.

Gatti, G., Brennan, M. J., \& Gardonio, P. (2007). Active damping of a beam using a physically collocated accelerometer and piezoelectric patch actuator. Journal of Sound and Vibration, 303, 798-813.

Gawronski, W. W. (1998). Dynamics and control of structures: A modal approach (1st ed.). New York: Springer-Verlag.

Gawronski, W. (2004). Advanced structural dynamics and active control of structures. New York: Springer. ISBN: 0387406492.

Ho, B. L., Kalman, R. E. (1965). Effective construction of linear statevariable models from input/output data. In 3rd annual Allerton conference on circuit and system theory (pp. 449-459).

Juang, J. N., \& Pappa, R. S. (1985). An eigensystem realization algorithm for modal parameter identification and modal reduction. Journal of Guidance, Control and Dynamics, 8(5), 620-627.
Juang, J. N., \& Wright, J. R. (1988). An eigensystem realization algorithm using data correlations (ERA/DC) for model parameter identification. Control Theory and Advanced Technology, 4(1), 5-44.

Lim, R. K., Phan, M. Q., \& Longman, R. W. (1998). State-space system identification with identified Hankel matrix. Department of Mechanical and Aerospace Engineering Technical Report No. 3045, Princeton University, Princeton, NJ.

Ljung, L. (1987). System identification: Theory for the user. Englewood Cliffs, NJ: Prentice Hall PTR.

Lus, H., Betti, R., \& Longman, R. W. (2002). Obtaining refined first order predictive models of linear structural systems. Earthquake Engineering and Structural Dynamics, 31, 1413-1440.

Monteiro, L. H. A. (2006). Sistemas Dinâmicos (2nd ed.). São Paulo, SP: Livraria da Física Editora.

Ogata, K. (2003). Engenharia de Controle Moderno (3a ed.). Rio de Janeiro, RJ: LTC- Livros Técnicos e Científicos Publisher S.A.

Phan, M. Q., Horta, L. G., Juang, J. N., \& Longman, R. W. (1992). Identification of linear systems by an asymptotically stable observer, NASA Langley Research Center Report L-16940. Hampton, VA: Langley Research Center.

Rezende, J. C. C., \& Borges, J. A. F. (2004). Comparação entre um Modelo de Elementos Finitos (MEF) e Análise Modal de uma Estrutura Veicular. Belém, PA: III Congresso Nacional de Engenharia Mecânica.

Santo, I. A. C. P. E. (2001). Modelação e Estimação de Parâmetros. Pedagogical aptitude test and technical capacity. Braga: Universidade do Minho.

Skogestad, S., \& Postlethwaite, I. (2005). Multivariable feedback control: Analysis and design (2nd ed.). New York: Wiley.

Zhang, J., He, L., Wang, E., \& Gao, R. (2008). A LQR controller design for active vibration control of flexible structures. In IEEE PacificAsia workshop on computational intelligence and industrial application. 